\title{
ß-Tantalum, a Better Candidate for Spin-to-Charge Conversion
}

\author{
David Magginetti, Kun Tian and Ashutosh Tiwari*
}

\begin{abstract}
Development of thermoelectric energy harvesting devices has hit a stumbling block due to the intrinsically linked electrical and thermal conductivities of materials. However, this field can still be improved by employing devices that take advantage of spin-based effects. A temperature gradient can be converted to a spin-polarized current in a ferrimagnetic insulator by the spin Seebeck effect (SSE), and that spin current can be converted to an electrical voltage in a heavy metal by the Inverse spin Hall effect (ISHE). Thus, the thermal energy capture and charge production steps can be separated into two distinct regions of the thermoelectric device, allowing separate tuning of electrical and thermal conductivities. The second step of this process, spin current to electrical voltage conversion, is controlled by the strength and sign of ISHE in the metal, and platinum has become the standard for this purpose. However, here we report a better candidate, $\beta$-Tantalum, which shows a spin Seebeck voltage approximately 4 times higher than that of $\mathrm{Pt}$ at room temperature. The temperature dependence of spin Seebeck in YIG/ $\beta$-Ta also closely follows that of YIG/Pt, consistent with magnon spin current theory. The sign of the spin Seebeck voltage in $\mathrm{Ta}$ found to be opposite to that of $\mathrm{Pt}$, making the two materials highly complementary for fabricating spintronics-based thermoelectric modules for practical applications.
\end{abstract}

Key words: D. energy harvesting; D. inverse spin Hall effect; A. spin Hall metals 


\section{Introduction}

The discovery of the spin Seebeck effect (SSE), the creation of a spin current in response to a temperature gradient applied across a ferromagnet [1], has opened up an important new field called spin caloritronics. While this has been a great advance for sourcing spin polarized currents in semiconductor channels, an additional benefit can also be derived in the field of energy harvesting. Spin polarization can be generated in a ferrimagnetic insulator, which can diffuse to adjacent layers, and be converted to a charge current by a metal exhibiting a large inverse spin Hall effect (ISHE) [1]. This bilayer structure presents an opportunity to improve waste heat energy harvesting as conventional thermoelectric materials have been greatly limited by the conflicting need to minimize thermal conductivity while maximizing electrical conductivity [2].

Equally important, as the need to optimize thermal and electrical conductivities, is the maximization of the signal transduction from thermal energy to spin current and then from spin current to electrical voltage. Yttrium iron garnet (YIG) has recently become the standard material for generating spin current [3-6] owing to its small coercivity and damping constant. For converting spin current into electrical voltage, metals exhibiting large ISHE are needed. The magnitude of a material's ISHE efficiency, enumerated by the spin Hall angle $\left(\theta_{\mathrm{SH}}\right)$, is highly dependent on the strength of spin orbit coupling in the material, and there has been a strong emphasis on studying heavy metals [7]. Platinum in particular has seen a great deal of study, both in terms of optimizing its thickness and structure to maximize SSE signal [8], and also for use in studying other parameters related to the Pt/YIG system [9].

While Pt has been invaluable for investigating the spin Seebeck effect, such singleminded focus on this material neglects the improvements that can be made by studying 
additional materials that can potentially exhibit a large ISHE effect. Among other heavy metals, the tetragonal $\beta$ phase of Ta was initially predicted to possess a large $\theta_{\mathrm{SH}}[10]$, and a subsequent experimental study of $\beta$-Ta showed that its Onsager reciprocal effect, the spin Hall effect, is large enough to switch the magnetization of a ferromagnetic thin film [11]. Prior work has also been done on testing the ISHE performance of $\beta$-Ta by spin pumping through thermal and microwave-based methods [12-14], but none have done a systematic study of how $\beta$-Ta performs as a spin-to-charge converter across a wide temperature range.

In this work we, for the first time, report the ISHE performance of $\beta$-Ta in an SSE device from room temperature down to $4 \mathrm{~K}$. In order to control for any possible variation in measurement conditions from study to study and to provide a baseline for performance, we tested devices in which a strip of $\beta$-Ta was deposited along with a strip of Pt on the same YIG substrate. By measurement of ISHE, we demonstrated excellent performance of $\beta$-Ta relative to Pt and showed the opposite signs of their $\theta_{\mathrm{SH}}$ values. Furthermore, measurement of the SSE signal from room temperature down to $4 \mathrm{~K}$ showed the similarity of the temperature dependence of these two materials. After comparing our results to past theoretical and experimental results, we briefly discussed the potential of $\beta$-Ta for fabricating practical spin-based thermoelectric devices.

\section{Experimental}

A schematic diagram of the samples investigated in this study is given in figure 1. Ta and Pt films measuring $3 \mathrm{~mm} \times 1 \mathrm{~mm}$ were deposited on a commercially-procured YIG film $(\sim 3 \mu \mathrm{m}$ thick) using DC magnetron sputtering. The deposition area for each strip was controlled using an aluminum shadow mask. The deposition was done at room temperature with an Argon pressure 
of $4 \mathrm{mTorr}$ for both metals. The films were separated by $1 \mathrm{~mm}$ and electrical measurements confirmed that there was no conductive pathway between them. Thickness and roughness of the films were determined by atomic force microscopy (AFM).

The sample was mounted in a closed cycle cryostat capable of cooling down to $4 \mathrm{~K}$. The sample was sandwiched between two thermally conducting polycrystalline AlN plates, and the temperature gradient was measured and maintained using two silicon diodes and two resistive wire heaters controlled by a Lakeshore 335 temperature controller. For more information about the experimental setup, see figure-S1 in supplementary information section. The base temperature was varied from $298 \mathrm{~K}$ down to $4 \mathrm{~K} . \Delta \mathrm{T}$ at each base temperature was varied from 0 to $10 \mathrm{~K}$. At each $\Delta \mathrm{T}$, magnetic field (B) was applied along the long axis of the YIG substrate and voltage developed across each metal strip was measured. Upon completion of the SSE measurements, the same sample was remounted in the cryostat to measure resistivity of the metal strips as a function of temperature in four-probe configuration.

\section{Results and Discussion}

AFM step profile experiments showed that the thicknesses for both Ta and Pt films deposited in our study were approximately $7 \mathrm{~nm}$. The films were quite smooth (figure 2), with rms roughness values of $0.2 \mathrm{~nm}, 0.8 \mathrm{~nm}$, and $0.2 \mathrm{~nm}$ for Ta and Pt and YIG, respectively. The electrical resistivity vs temperature measurements showed that $\mathrm{Ta}$ exhibits a negative temperature coefficient of resistivity (TCR) with a room temperature resistivity value of $\sim 250 \mu$ $\Omega \mathrm{cm}$ (see figure 3(a)). Relatively large value of electrical resistivity and negative TCR are considered as the fingerprint characteristics of $\beta$-Ta [15]. Pt's resistivity showed a positive TCR similar to conventional metallic samples (see figure 3(b)). 
For measuring the ISHE response of the samples, heat was applied from the top of the sample, and magnetic field was applied along the length of YIG. Figures 4(a) and (b) show the experimentally measured $\mathrm{V}_{\mathrm{SSE}}$ vs. B data recorded from $\beta$-Ta and Pt strips for different $\Delta \mathrm{T}$ values. Measurements taken at both positive and negative fields allow the elimination of spurious effects which might arise due to conventional Seebeck effect, and the measurements at $\Delta \mathrm{T}=0 \mathrm{~K}$ show a change in sign due to parasitic heat fluxes. The absolute value of $\mathrm{V}_{\text {SSE }}$ is calculated as the half of the difference between the mean of the measured voltages at positive magnetic field and the mean of the voltages at negative field and is plotted against $\Delta \mathrm{T}$ for $\beta$-Ta and Pt in figures 4(c) and $(d)$, respectively. The sign of SSE signal $\left(\mathrm{V}_{\mathrm{SSE}}\right)$ is governed by the spatial direction of spin current, direction of thee spin polarization vector and the sign of the spin Hall angle $\left(\theta_{\mathrm{SH}}\right)$ of the top metallic layer $[5,16]$. Under the identical conditions of spin current polarization and direction, metals with opposite signs of $\theta_{\mathrm{SH}}$ should give $\mathrm{V}_{\mathrm{SSE}}$ voltages with opposite polarities. The opposite signs of the observed voltages as a function of magnetic field show the opposite signs of the $\theta_{\mathrm{SH}}$ for $\beta$-Ta and Pt. Both the plots show linear trends from which room temperature spin Seebeck coefficient $\left(\mathrm{S}_{\mathrm{SSE}}=\mathrm{V}_{\mathrm{SSE}} / \Delta \mathrm{T}\right)$ values were calculated. The room temperature $\mathrm{S}_{\mathrm{SSE}}$ of $\beta$-Ta was $123 \mathrm{nV} / \mathrm{K}$ which was about four times larger than the corresponding vale of $-28 \mathrm{nV} / \mathrm{K}$ for Pt. This much larger $S_{S S E}$ value for $\beta$-Ta continued down to a base temperature of approximately $50 \mathrm{~K}$ (figure 5).

The observed larger value of $\mathrm{V}_{\text {SSE }}$ for $\beta$-Ta can be understood by looking into the theoretical relationship for $\mathrm{V}_{\mathrm{SSE}}[7,17-19]$ :

$V S S E \propto \rho \theta S H g \uparrow \downarrow \lambda S \tanh t 2 \lambda S$ 
where $\lambda S, \rho, \theta S H$, and $g \uparrow \downarrow$ are the spin diffusion length of the metal, the resistivity of the metal, the metal's spin hall angle, and the spin mixing conductance between the metal and the ferrimagnet, respectively. Thickness is denoted by $t$, which is the same for both materials. $\beta$-Ta is known to have a larger $\theta S H$ and, as we have shown in figure 3, larger $\rho$. Previous studies on $\beta$ Ta have shown that its spin diffusion length is approximately the same as Pt's, however the spin mixing conductance of the former is much smaller than the latter [12]. The smaller spin mixing conductance in YIG/ $\beta$-Ta likely accounts for seeing only a four-fold increase in SSE signal vs. YIG/Pt, while the product of $\theta S H$ and $\rho$ for $\beta$-Ta should be greater than 20 times that of Pt.

As reported previously $[8,16,20]$, materials with large negative spin Hall angle such as $\beta$ Ta are potentially of great use as a complement to Pt for energy harvesting using a longitudinal SSE geometry. For example, in conventional thermoelectric modules, alternating cells of n- and p-type materials are connected in an optimized configuration to result in the maximum power output. The same can be done in the case of SSE-based devices by using alternating cells of YIG/Pt and YIG/Ta. With the extensive study that has now been performed on both these materials, Pt and Ta are good candidates for investigating the performance of a more practical device. A schematic diagram of the proposed spintronics-based thermoelectric module is illustrated in figure-S2 in the supplementary information section.

In addition to finding materials that show the largest possible $\mathrm{V}_{\mathrm{SSE}}$ voltages in response to applied temperature differences, it is equally important to understand the underlying mechanisms and how the experimental results correlate with theoretical predictions. For this we performed temperature dependent $V_{\text {SSE }}$ measurements over the temperature range 4-298 K. Early theoretical studies by Xiao et al. [17,21] predicted that $\mathrm{V}_{\mathrm{SSE}}$ in longitudinal YIG/Pt device should 
monotonically decrease with decreasing base temperature. This prediction was based on the assumption that the population of magnons in YIG decreases on decreasing the temperature. However, in a more recent study Rezende et al. [19] predicted and experimentally showed using that $\mathrm{V}_{\mathrm{SSE}}$ should exhibit a maximum at low temperatures. The low temperature maximum in $\mathrm{V}_{\mathrm{SSE}}$ was explained to be due to the competition between the magnon population, which decreases with reduced temperature, and magnon lifetime, which increases with decreasing temperature [19]. Our results for both YIG/Pt as well as YIG/Ta showed maxima at around $200 \mathrm{~K}$, in good agreement with this theory and a study on the temperature dependence of $\mathrm{V}_{\text {SSE }}$ in YIG/Pt [19]. Moreover, the similar temperature dependence for both YIG/Ta and YIG/Pt supports the theory that the paramagnetic metal does not make any significant contribution to the temperature dependence of the signal.

\section{Conclusions}

In summary, by controlled measurement of $\beta-\mathrm{Ta}$ and Pt thin films deposited on a ferrimagnetic insulator, YIG, we have shown a great enhancement in the thermal to electrical energy conversion using $\beta$-Ta as the ISHE material. At room temperature the $\mathrm{V}_{\text {SSE }}$ signal generated by $\beta-\mathrm{Ta}$ is 4 times larger than that of $\mathrm{Pt}$ and at least 3 times larger at temperatures down to $50 \mathrm{~K}$. Comparison of these temperature-dependent results indicates excellent agreement with recent theoretical predictions for longitudinal SSE and provides further validation to the theory of Rezende et al. The large ISHE signal of $\beta$-Ta along with its negative $\theta_{\mathrm{SH}}$ makes it an ideal candidate for designing next-generation spintronics-based thermoelectric modules.

\section{Acknowledgement:}

Research was supported by U.S. National Science Foundation through Award No. 1407650 and 1121252 (MRSEC). 


\section{References:}

1. K. Uchida, S. Takahashi, K. Harii, J. Ieda, W. Koshibae, K. Ando, S. Maekawa, E. Saitoh, Nature 455 (2008) 778-781.

2. G.J. Snyder, E.S. Toberer, Nat. Mater. 7 (2008) 105-114.

3. K.I. Uchida, H. Adachi, T. Ota, H. Nakayama, S. Maekawa, E. Saitoh, Appl. Phys. Lett. 97 (2010) 172505.

4. M. Schreier, G.E.W. Bauer, V. Vasyuchka, J. Flipse, K. Uchida, V. Lauer, A. Chumak, A. Serga, S. Daimon, T. Kikkawa, B.J. Van Wees, B. Hillebrands, R. Gross, S.T.B. Goennenwein, J. Phys. D. Appl. Phys. 48 (2014) 025001.

5. G. Siegel, M.C. Prestgard, S. Teng, A. Tiwari, Sci. Rep. 4 (2014) 4429.

6. S.R. Boona, R.C. Myers, J.P. Heremans, Energy Environ. Sci. 7 (2014) 885.

7. A. Hoffmann, IEEE Trans. Magn. 49 (2013) 5172-5193.

8. Y. Saiga, K. Mizunuma, Y. Kono, J.C. Ryu, H. Ono, M. Kohda, E. Okuno, Appl. Phys. Express 7 (2014) 093001.

9. A. Kehlberger, U. Ritzmann, D. Hinzke, E.J. Guo, J. Cramer, G. Jakob, M.C. Onbasli, D.H. Kim, C.A. Ross, M.B. Jungfleisch, B. Hillebrands, U. Nowak, M. Kläui, Phys. Rev. Lett. 115 (2015) 096602.

10. T. Tanaka, H. Kontani, M. Naito, T. Naito, D.S. Hirashima, K. Yamada, J. Inoue, Phys. Rev. B - Condens. Matter Mater. Phys. 77 (2008) 165117.

11. L. Liu, C.-F. Pai, Y. Li, H.W. Tseng, D.C. Ralph, R.A. Buhrman, Science 336 (2012) $555-558$.

12. C. Hahn, G. De Loubens, O. Klein, M. Viret, V. V. Naletov, J. Ben Youssef, Phys. Rev. B - Condens. Matter Mater. Phys. 87 (2013) 174417.

13. K.-D. Lee, D.-J. Kim, H. Yeon Lee, S.-H. Kim, J.-H. Lee, K.-M. Lee, J.-R. Jeong, K.-S. Lee, H.-S. Song, J.-W. Sohn, S.-C. Shin, B.-G. Park, Sci. Rep. 5 (2015) 10249.

14. N. Vlietstra, J. Shan, B.J. Van Wees, M. Isasa, F. Casanova, J. Ben Youssef, Phys. Rev. B - Condens. Matter Mater. Phys. 90 (2014) 174436.

15. N. Schwartz, W.A. Reed, P. Polash, M.H. Read, Thin Solid Films 14 (1972) 333-246.

16. K. Uchida, M. Ishida, T. Kikkawa, A. Kirihara, T. Murakami, E. Saitoh, J. Phys. Condens. Matter 26 (2014) 343202.

17. J. Xiao, G.E.W. Bauer, K.C. Uchida, E. Saitoh, S. Maekawa, Phys. Rev. B - Condens. Matter Mater. Phys. 81 (2010) 214418.

18. H. Adachi, K. Uchida, E. Saitoh, J. Ohe, S. Takahashi, S. Maekawa, Appl. Phys. Lett. 97 (2010) 252506. 
19. S. M. Rezende, R. L. Rodríguez-Suárez, R. O. Cunha, A. R. Rodrigues, F. L. A. Machado, G. A. Fonseca Guerra, J. C. Lopez Ortiz, and A. Azevedo, Phys. Rev. B Condens. Matter Mater. Phys. 89 (2014) 014416.

20. A. Kirihara, K Uchida, Y. Kajiwara, M. Ishida, Y Nakamura, T. Manako, E. Saitoh, and S. Yorozu, Nat. Mat. 11 (2012) 686.

21. S. Wang, L. Zou, X. Zhang, J. Cai, S. Wang, B. Shen, J. Sun, Nanoscale 7 (2015) 1781217819. 


\section{Figure captions:}

Figure 1: Schematic diagram of the SSE device. With top heating and an in-plane positive magnetic field, a positive voltage is measured across Ta and a negative voltage is measured across Pt.

Figure 2: AFM micrographs of YIG (a), Pt/YIG (b), and Ta/YIG (c). The rms roughness values for YIG, Pt, and $\beta$-Ta were $0.2 \mathrm{~nm}, 0.8 \mathrm{~nm}$, and $0.2 \mathrm{~nm}$, respectively.

Figure 3: Resistivity vs. temperature for $\beta$-Ta (a) and $\mathrm{Pt}(\mathrm{b})$.

Figure 4: $\mathrm{V}_{\mathrm{SSE}}$ as a function of temperature difference at $298 \mathrm{~K}$. Voltage vs. magnetic field at varying temperature differentials is plotted for $\beta$ - Ta (a) and $\mathrm{Pt}(\mathrm{b})$. The $\mathrm{V}_{\mathrm{SSE}}$ values which are extracted from this data are plotted against temperature differential for $\beta$-Ta $(c)$ and $\mathrm{Pt}(\mathrm{d})$.

Figure 5: Spin Seebeck coefficient, $\mathrm{S}_{\mathrm{SSE}}$, vs. base temperature for $\beta$-Ta (a) and $\mathrm{Pt}(\mathrm{b})$. 


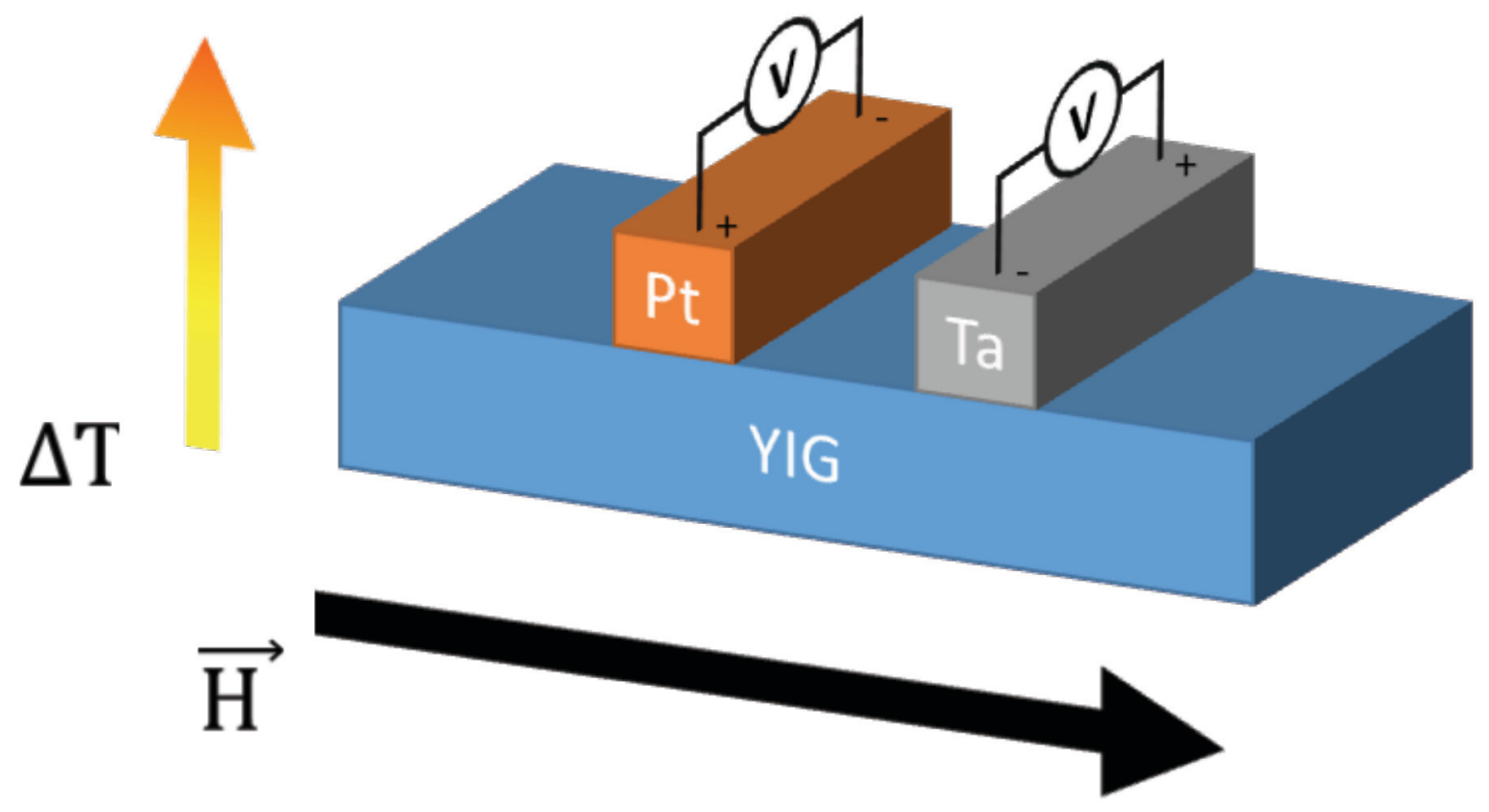

Fig. 1

Magginetti et al. 

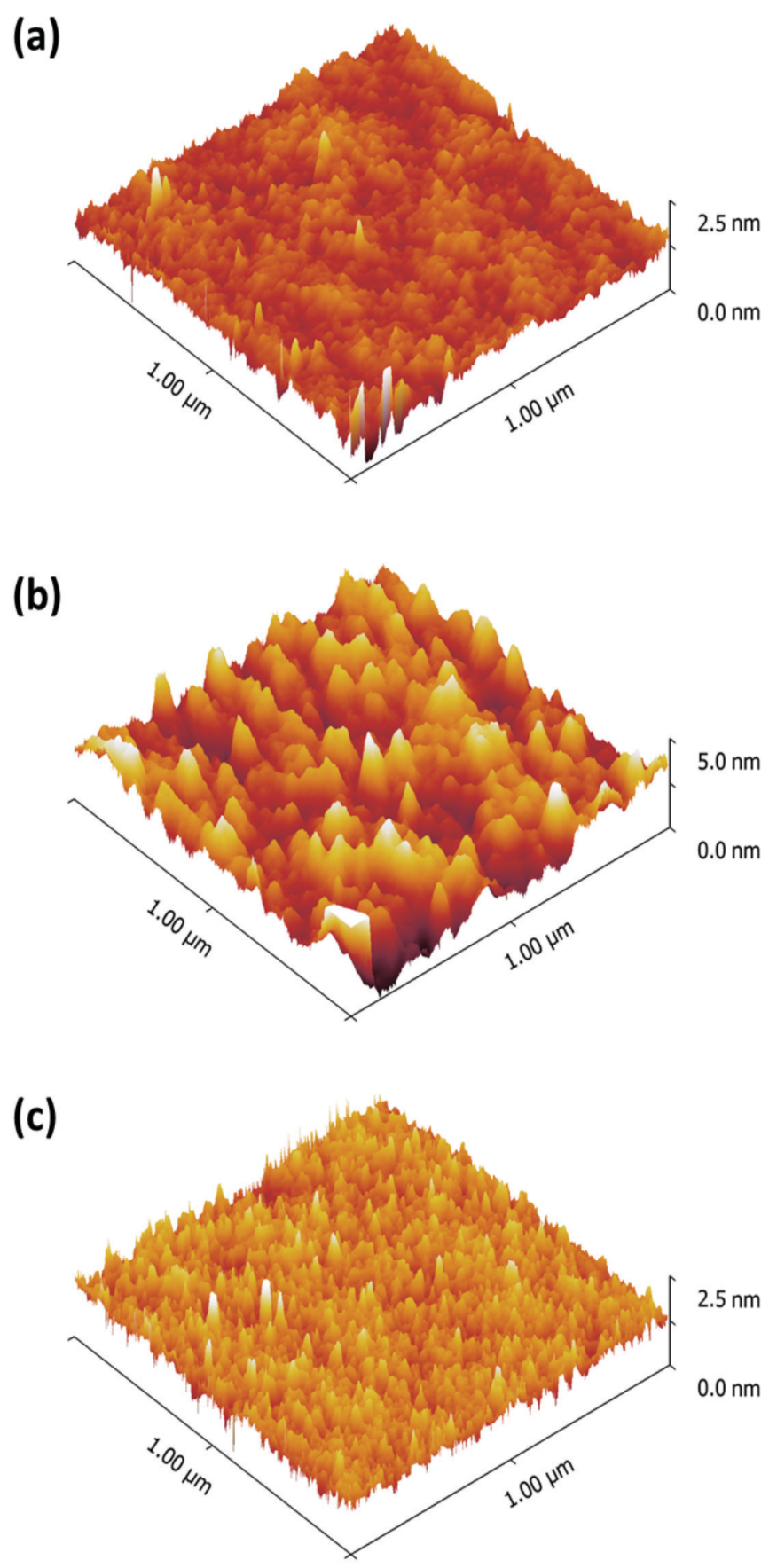

Fig. 2

Magginetti et al. 


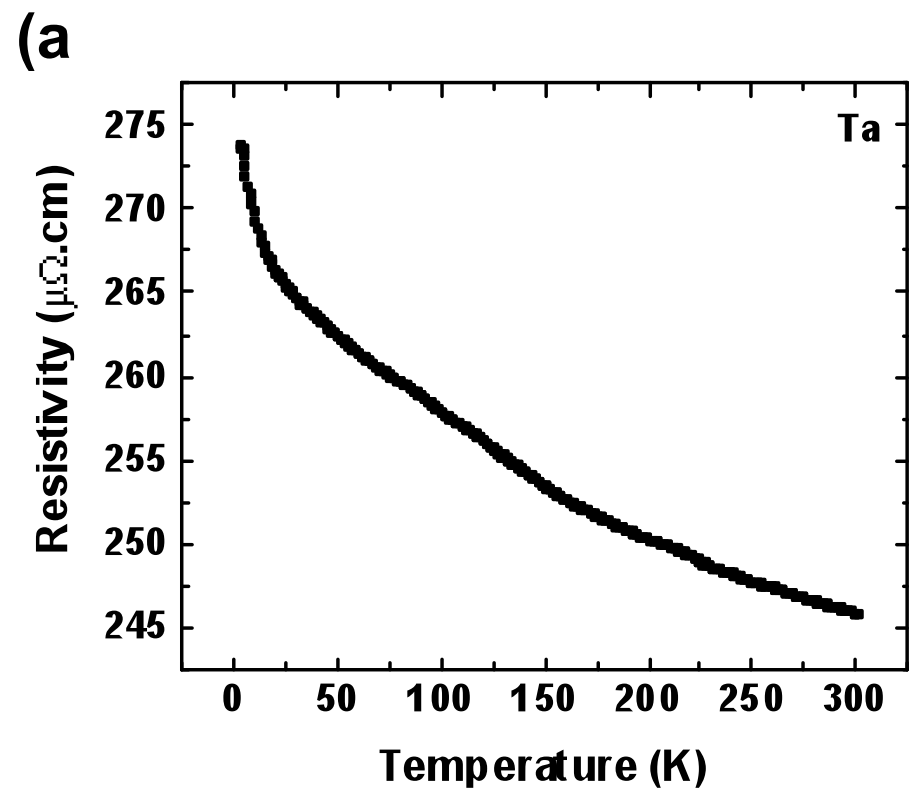

(b

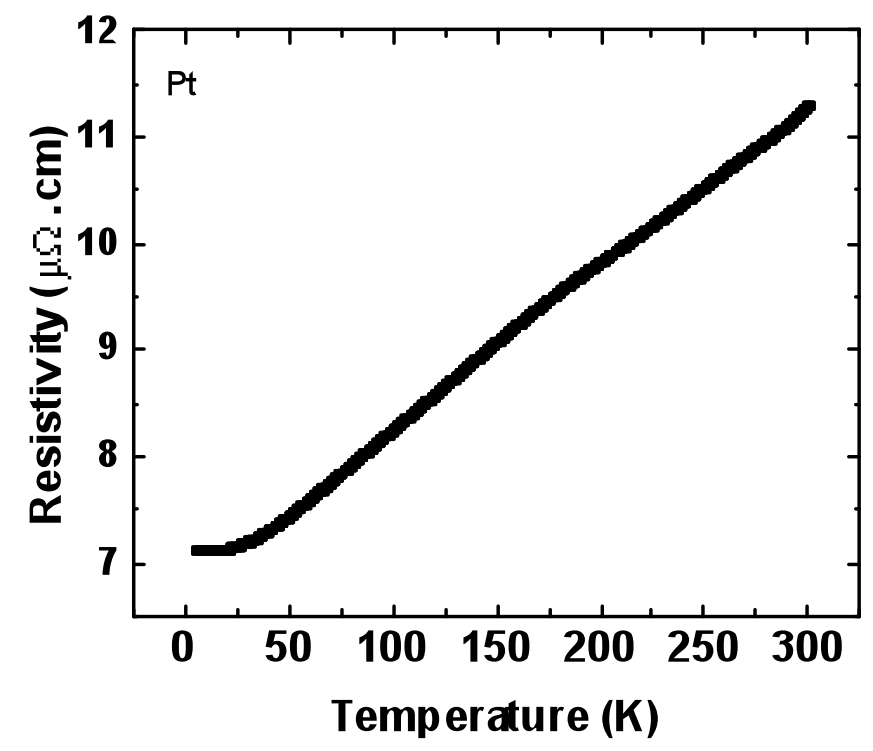

Fig. 3 
(a)

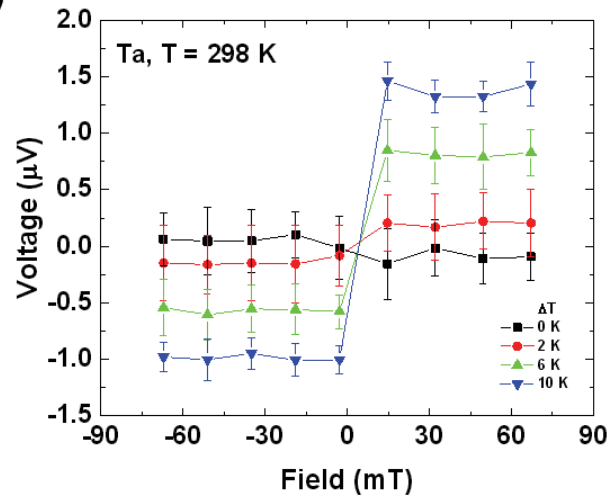

(c)

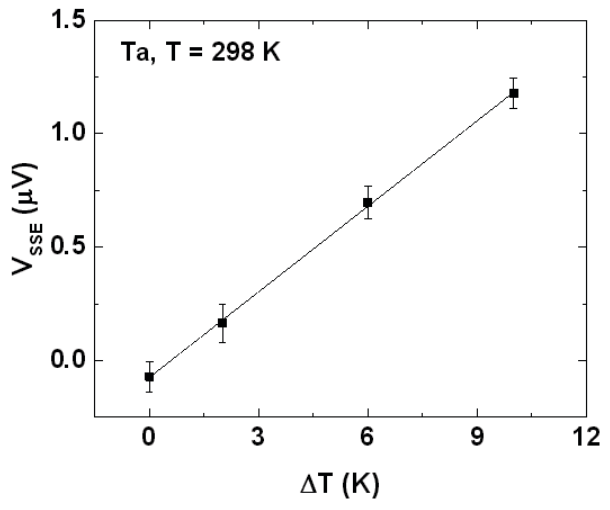

(b)
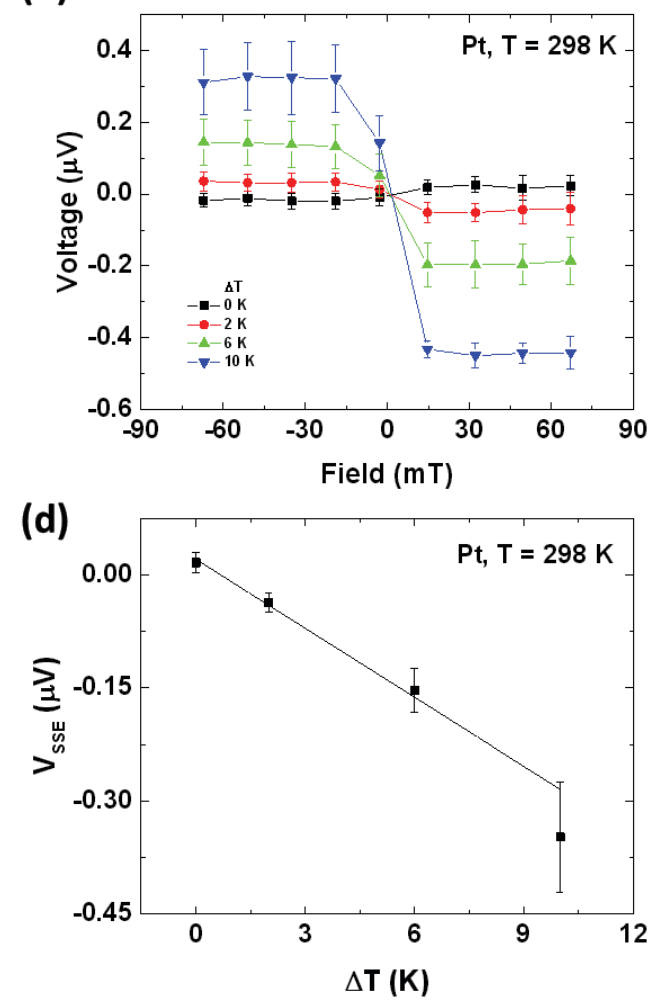

Fig. 4 
(a)

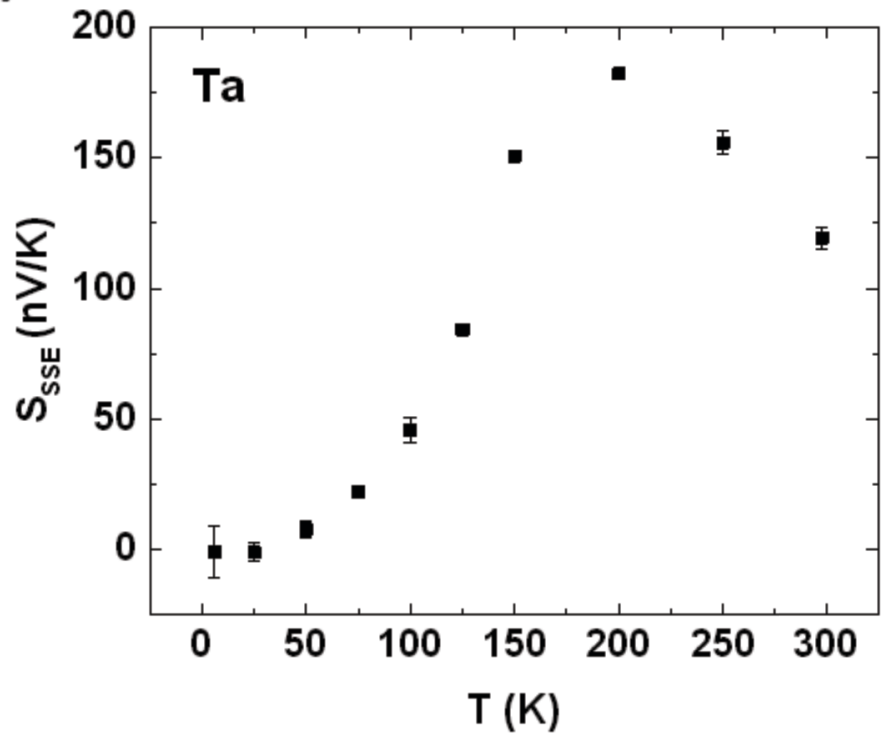

(b)

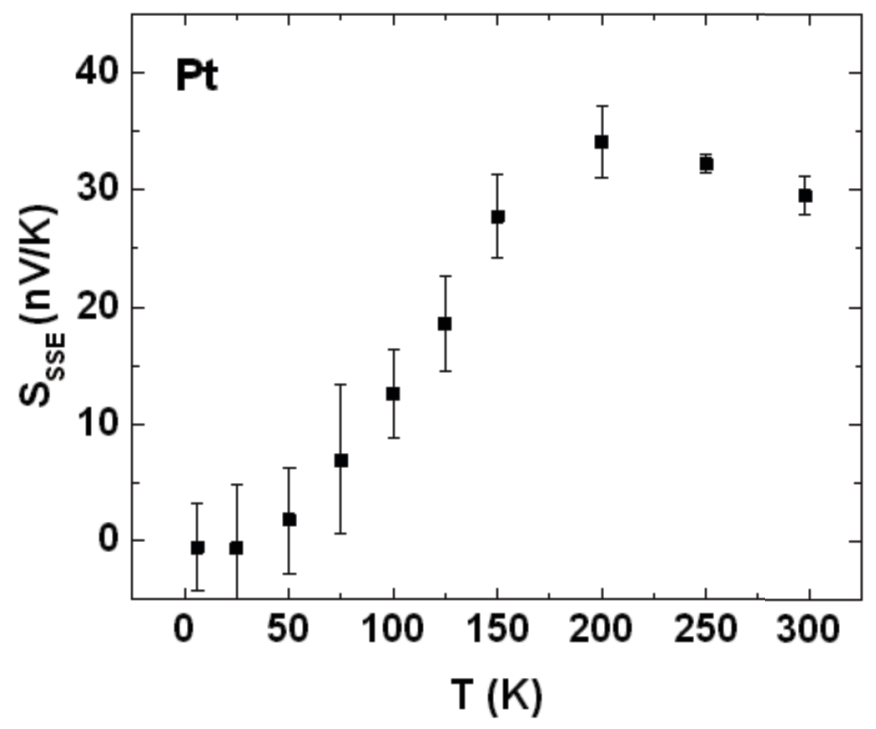

Fig. 5

Magginetti et al. 